\title{
A New Martingale Approach to Kalman Filtering
}

\section{ARUNABHA BAGCHI}

Department of Applied Mathematics, Twente University of Technology, Enschede, Postbus 217, The Netherlands

Communicated by Karl-Johan Ástrom

\section{ABSTRACT}

A new derivation of continuous-time Kalman Filter equations is presented. The underlying idea has been previously used to derive the smoothing equations. A unified approach to filtering and smoothing problems has thus been achieved.

\section{INTRODUCTION}

Recently many rigorous derivations of continuous-time Kalman Filter equations have been obtained $[1,2,3]$. The most general nonlinear problem has been studied in [3] but its specialization to the linear case obscures the simplicity of the linear problem. An elegant proof in the linear case has been proposed in [1] which exploits a result on the estimation of one martingale from another. As pointed out in [4], the "state martingale" used in [1] does not yield the smoothing equations and a different martingale has, therefore, been proposed. This paper shows that the same martingale can be used to derive the filtering equations also, thus unifying the martingale technique initiated in [1] to derive both the filtering and smoothing equations.

\section{PROBLEM FORMULATION}

Let us consider the linear stochastic equations (continuous version)

$$
\begin{aligned}
& x(t ; \omega)=\int_{o}^{t} A(\sigma) x(\sigma ; \omega) d \sigma+\int_{o}^{t} B(\sigma) d W(\sigma ; \omega)(2 \cdot 1) \\
& y(t ; \omega)=\int_{o}^{t} C(\sigma) x(\sigma ; \omega) d \sigma+\int_{o}^{t} D(\sigma) d W(\sigma ; \omega)(2 \cdot 2)
\end{aligned}
$$

for $0 \leqslant t \leqslant T$ where $x(t ; \omega)$ and $y(t ; \omega)$ take values in $n$ - and $m$-dimensional Euclidean spaces $R^{n}$ and $R^{m}$, respectively, $W(t ; \omega)$ is a $p$-dimensional Wiener process, and $A(t), B(t), C(t)$, and $D(t)$ are appropriate dimensional matrixvalued functions. Assume that these coefficient functions are all continuous and $D(t) D(t)^{*}>0$ on the interval [0.T] of interest, where * stands for the transpose. 
Let $\beta(s)$ be the smallest $\sigma$-algebra generated by the process $y(\sigma ; \omega), 0 \leqslant \sigma \leqslant s$ completed with respect to sets of measure 0 and $\beta(s-)$ the smallest $\sigma$-algebra generated by the process $y(\sigma ; \omega), 0 \leqslant \sigma<s$ completed with respect to sets of measure 0 . Then since $y(t ; \omega)$ is continuous in $t$ with probability one, $\beta(s)=$ $\beta(s-)$.

Let $\hat{x}(t \mid s)=E[x(t) \mid \beta(s)]$. Then it is well known $[5, \mathrm{p}$. 44] that $\hat{x}(t)=$ $\hat{x}(t \mid t)$ is the best minimum variance estimate of $x(t)$ based on the observation $y(\sigma ; \omega), 0 \leqslant \sigma \leqslant t$ and is called the filtered estimate of $x(t)$. Since $\hat{x}(t \mid s)$ is a martingale in $s$ for fixed $t$ we have from $[6, \mathrm{p} .121]$

$$
\hat{x}(t \mid s)=\int_{o}^{s} \gamma_{12}(\tau) d Z_{0}(\tau ; \omega)
$$

where $Z_{o}(t ; \omega)$, the so-called innovation process, is defined as

$$
Z_{o}(t ; \omega)=y(t ; \omega)-\int_{o}^{t} C(\sigma) \hat{x}(\sigma ; \omega) d \sigma
$$

and

$$
\gamma_{12}(\tau)=P_{12}(\tau) P_{22}(\tau)^{-1}
$$

where

$$
\begin{aligned}
& P_{12}(\tau)=\lim _{\Delta \rightarrow o} \frac{1}{\Delta} E\left[(\hat{x}(t \mid \tau+\Delta)-\hat{x}(t \mid \tau))\left(Z_{o}(\tau+\Delta)-Z_{o}(\tau)\right)^{*} \mid \beta(\tau)\right], \\
& P_{22}(\tau)=\lim _{\Delta \rightarrow o} \frac{1}{\Delta} E\left[\left(\int_{\tau}^{\tau+\Delta} d Z_{o}(\sigma ; \omega)\right)\left(\int_{\tau}^{\tau+\Delta} d Z_{o}(\sigma ; \omega)\right)^{*} \mid \beta(\tau)\right] .
\end{aligned}
$$

\section{FILTERING EQUATIONS}

Let us consider (2.3) for $s<t$. From [6,p. 127], for any $\tau>0$

$$
P_{22}(\tau)=D(\tau) D(\tau)^{*}
$$

while for $\tau<t$

$$
P_{12}(\tau)=\lim _{\Delta \rightarrow o} \frac{1}{\Delta} E\left[\left(\hat{x}(t \mid \tau+\Delta)-\hat{x}(t \mid \tau)\left(Z_{o}(\tau+\Delta)-Z_{o}(\tau)\right)^{*} \mid \beta(\tau)\right]\right.
$$

where $\tau$ and $\tau+\Delta$ are both less than $t$. Now

$$
x(t)=\Phi(t, \tau+\Delta) x(\tau+\Delta)+\int_{\tau+\Delta}^{t} \Phi(t, \sigma) d W(\sigma)
$$

where $\Phi(t, \tau)$ is the fundamental matrix of dimension $n \times n$ satisfying 


$$
\frac{d \Phi(t, \tau)}{d t}=A(t) \Phi(t, \tau) \quad \Phi(\tau, \tau)=I
$$

Hence

$$
E[x(t) \mid \beta(\tau+\Delta)]=\Phi(t, \tau+\Delta) \hat{x}(\tau+\Delta)
$$

and

$$
E[x(t) \mid \beta(\tau)]=\Phi(t, \tau) \hat{x}(\tau)
$$

With this, we have

$$
\begin{aligned}
& P_{12}(\tau)=\lim _{\Delta \rightarrow o} \frac{1}{\Delta} E[(\Phi(t, \tau+\Delta) \hat{x}(\tau+\Delta) \\
& \left.-\Phi(t, \tau) \hat{x}(\tau)\left(Z_{o}(\tau+\Delta)-Z_{o}(\tau)\right)^{*} \mid \beta(\tau)\right] \\
& =\Phi(t, \tau) \lim _{\Delta \rightarrow o} \frac{1}{\Delta} E\left[(\Phi(\tau, \tau+\Delta) \hat{x}(\tau+\Delta)-\hat{x}(\tau))\left(Z_{o}(\tau+\Delta)-Z_{o}(\tau)\right)^{*} \mid \beta(\tau)\right] \\
& =\Phi(t, \tau) \lim _{\Delta \rightarrow o} \frac{1}{\Delta} E[\{(\Phi(\tau, \tau+\Delta)-I) \hat{x}(\tau+\Delta)+\hat{x}(\tau+\Delta)-\hat{x}(\tau)\} \\
& =\Phi(t, \tau) \lim _{\Delta \rightarrow o} \frac{\Phi(\tau, \tau+\Delta)-I}{\Delta} E\left[\hat{x}(\tau+\Delta)\left(Z_{o}(\tau+\Delta)-Z_{o}(\tau)\right)^{*} \mid \beta(\tau)\right] \\
& +\Phi(t, \tau) \lim _{\Delta \rightarrow o} \frac{1}{\Delta} E\left[(\hat{x}(\tau+\Delta)-\hat{x}(\tau))\left(Z_{o}(\tau+\Delta)-Z_{o}(\tau)\right)^{*} \mid \beta(\tau)\right] \\
& =\Phi(t, \tau) \lim _{\Delta \rightarrow o} \frac{1}{\Delta} E\left[(\hat{x}(\tau+\Delta)-\hat{x}(\tau))\left(Z_{o}(\tau+\Delta)-Z_{o}(\tau)\right)^{*} \mid \beta(\tau)\right]
\end{aligned}
$$

since

$$
\lim _{\Delta \rightarrow 0} \frac{\Phi(\tau, \tau+\Delta)-1}{\Delta}=-A(\tau)
$$

exists and

$$
\lim _{\Delta \rightarrow o} E\left[\hat{x}(\tau+\Delta)\left(Z_{o}(\tau+\Delta)-Z_{o}(\tau)\right) * \mid \beta(\tau)\right]=0
$$

in $L_{1}$ sense. 
Let us define the error $e(t)=x(t)-\hat{x}(t)$. Then

$$
\begin{aligned}
& \hat{x}(\tau+\Delta)=x(\tau+\Delta)-e(\tau+\Delta) \\
& =\int_{o}^{\tau+\Delta} A(\sigma) x(\sigma) d \sigma+\int_{o}^{\tau+\Delta} B(\sigma) d W(\sigma)-e(\tau+\Delta)
\end{aligned}
$$

and so

$\hat{x}(\tau+\Delta)-\hat{x}(\tau)=e(\tau)-e(\tau+\Delta)+\int_{\tau}^{\tau+\Delta} A(\sigma) x(\sigma) d \sigma+\int_{\tau}^{\tau+\Delta} B(\sigma) d W(\sigma)$.

Let $P(t ; \tau)=E\left[e(t ; \omega) e(\tau ; \omega)^{*}\right]$. Then

$$
P_{12}(\tau)=\lim _{\Delta \rightarrow o} \frac{1}{\Delta} E[(e(\tau)-e(\tau+\Delta)+
$$

$\left.\left.\int_{\tau}^{\tau+\Delta} A(\sigma) x(\sigma) d \sigma+\int_{\tau}^{\tau+\Delta} B(\sigma) d W(\sigma)\right) \times\left(Z_{o}(\tau+\Delta)-Z_{o}(\tau)\right) * \mid B(\tau)\right]$

Now for any $\Delta>0, e(\tau+\Delta ; \omega)$ is uncorrelated with $y(\sigma ; \omega), \sigma \leqslant \tau+\Delta$ and hence with $Z_{o}(\sigma ; \omega), \sigma \leqslant \tau+\Delta$. It is also uncorrelated with (and hence independent of) the random variables generating $\beta(\tau)$. Hence

$$
E\left(e((\tau+\Delta) ; \omega)\left(\int_{\tau}^{\tau+\Delta} d Z_{o}(\sigma ; \omega)\right)^{*} \mid \beta(\tau)\right)=0
$$

Furthermore, we have the following $[6$, p. 129]:

$$
\begin{aligned}
& E\left(e(\tau)\left(\int_{\tau}^{\tau+\Delta} d Z_{o}(\sigma ; \omega)\right)^{*} \mid \beta(\tau)\right)=\int_{\tau}^{\tau+\Delta} P(\tau, \sigma) C(\sigma)^{*} d \sigma \\
& \mid E\left(\left(\int_{\tau}^{\tau+\Delta} A(\sigma) x(\sigma) d \sigma\right)\left(\int_{\tau}^{\tau+\Delta} d Z_{o}(\sigma ; \omega)\right)^{*} \mid=O\left(\mid \Delta \beta^{\beta / 2}\right)\right. \\
& E\left(\left(\int_{\tau}^{\tau+\Delta_{B}} B(\sigma) d W(\sigma ; \omega)\right)\left(\int_{\tau}^{\tau+\Delta} d Z_{o}(\sigma ; \omega)\right)^{*} \mid \beta(\tau)\right)=\int_{\tau}^{\tau+\Delta} B(\sigma) D(\sigma)^{*} \\
& d \sigma+O\left(\Delta^{3 / 2}\right)
\end{aligned}
$$

So

$$
P_{12}(\tau)=P(\tau) C(\tau)^{*}+B(\tau) D(\tau)^{*}
$$

where 


$$
P(\tau)=P(\tau, \tau)
$$

Thus we finally get, for $s<t$,

$$
\hat{x}(t \mid s)=\int_{o}^{s} \Phi(t, \tau)\left[P(\tau) C(\tau)^{*}+B(\tau) D(\tau)^{*}\right]\left(D(\tau) D(\tau)^{*}\right)^{-1} d Z_{o}(\tau)
$$

Now $\hat{x}(t \mid s)$ being a Martingale in $s$ for fixed $t$, we have from Doob [7, Theorem 4.3, p. 355]

$$
\lim _{s \rightarrow t_{-}} x(t \mid s)=E\left[x(t) \mid \beta\left(t_{-}\right)\right]=E[x(t) \mid \beta(t)]=\hat{x}(t)
$$

Hence taking limit in (3.1) as $s \rightarrow t-$, we get

$$
\hat{x}(t)=\int_{o}^{t} \Phi(t, \tau)\left[P(\tau) C(\tau)^{*}+B(\tau) D(\tau)^{*}\right]\left(D(\tau) D(\tau)^{*}\right)^{-1} d Z_{o}(\tau)
$$

or, writing

$$
K(t)=\left[P(t) C(t)^{*}+B(t) D(t)^{*}\right]\left(D(t) D(t)^{*}\right)^{-1},
$$

$\hat{x}(t)$ is the solution of the stochastic integral equation

$$
\hat{x}(t)=\int_{o}^{t} A \hat{x}(s) d s+\int_{o}^{t} K(s)[d y(s)-\mathrm{C}(s) \hat{x}(s) d s](3 \cdot 2)
$$

and $P(t)$, the error covariance matrix that appears in $K(t)$, satisfies the wellknown matrix Ricatti equation [6, Corollary 2, p. 137]

$$
\begin{aligned}
& \qquad \frac{d}{d t} P(t)=A(t) P(t)+P(t) A(t)^{*}+B(t) B(t)^{*} \\
& -\left[P(t) C(t)^{*}+B(t) D(t)^{*}\right]\left(D(t) D(t)^{*}\right)^{-1}\left[C(t) P(t)+D(t) B(t)^{*}\right](3 \cdot 3) \\
& \text { with } P(O)=O .
\end{aligned}
$$

\section{CONCLUSION}

A new derivation of linear recursive filtering equations is presented. This, with an earlier paper [4], enables us to give a unified rigorous approach to linear filtering and smoothing problems in continuous-time dynamical systems. 


\section{REFERENCES}

1. A. V. Balakrishnan, A Martingale approach to linear recursive state estimation, SIAM J. Control 10, No. 4, 754-756 (1972).

2. T. Kailath, A note on least squares estimations by the innovation method, SIAM J. Control 10, No. 3, 477-486 (1972).

3. M. Fujisaki, G. Kallianpur, and H. Kunita, Stochastic differential equations for the nonlinear filtering problem, Osaka J. Math. 9, No. 1, 19-40 (1972).

4. A. Bagchi, A Martingale approach to continuous time linear smoothing, SIAM J. Appl. Math. 10, No. 2, 276-281 (1975).

5. R. S. Bucy and P. D. Joseph, Filtering for Stochastic Processes with Applications To Guidance, Wiley-Interscience, New York, 1968.

6. A. V. Balakrishnan, Stochastic Differential Systems I, Lecture Notes in Economics and Mathematical Systems, No. 84, Springer-Verlag, Berlin, 1973.

7. J. L. Doob, Stochastic Processes, John Wiley, New York, 1953.

Received December, 1974 